\title{
The Dynamical Gas Evolution of Low-and Intermediate-Mass Stars: PN Formation
}

\author{
Eva Villaver \\ Space Telescope Science Institute, 3700 San Martin Drive, Baltimore, \\ $M D$ 21218, USA \\ Arturo Manchado \\ Instituto de Astrofísica de Canarias, c/ Via Láctea s/n 38205 La \\ Laguna (Tenerife), Spain \\ Guillermo García-Segura \\ Instituto de Astronomía-Universidad Nacional Autónoma de México, \\ Apartado Postal 877, Ensenada, 22830 Baja California, México
}

\begin{abstract}
Planetary nebula (PN) formation is intimately linked to stellar evolution. The stellar wind during the AGB and post-AGB stages depends on the progenitor mass and determines, together with the radiation field of the star, the structure and evolution of PNe. We summarize the results of the $\mathrm{PhD}$ thesis of $\mathrm{E}$. Villaver, where we combined the results of stellar evolution with a numerical code to study the PN formation process.
\end{abstract}

We have approached the problem of planetary nebula (PN) formation in two ways. First, we observe the properties of a large sample (15) of PNe which morphologically show multiple shells and study the kinematics of the different shells by obtaining and analyzing high-resolution, spatially resolved echelle spectra. PNe with attached and detached shells are included in the sample in order to allow a comparison of the kinematic properties of the two subclasses of multiple shell PNe. Second, we have followed the evolution of the circumstellar gas from the thermal pulse phase during the AGB stage to PN formation. Our hydrodynamical simulations use realistic winds obtained from stellar evolution calculations. In an attempt to obtain a complete picture for the PN scenario, we investigate the evolution of the gas ejected for the whole range of stellar masses, which, according to stellar evolutionary theory, gives rise to $\mathrm{PN}$ formation. Six models with masses of $1,1.5,2,2.5,3.5$ and $5 M_{\odot}$ have been used. We also study the effects of different ISM environments.

We investigate the dynamical evolution of the structures generated by the stellar wind during the AGB phase, the transition time and finally the PN stages. We find that the circumstellar gas evolution during the AGB phase is highly non-linear (snapshots during the AGB evolution are shown in Fig. 1). We show how variations in the wind that are associated with the thermal pulses do not remain recorded in the density or velocity structure of the gas because the wind variations lead to the formation of transient shells with an average lifetime 


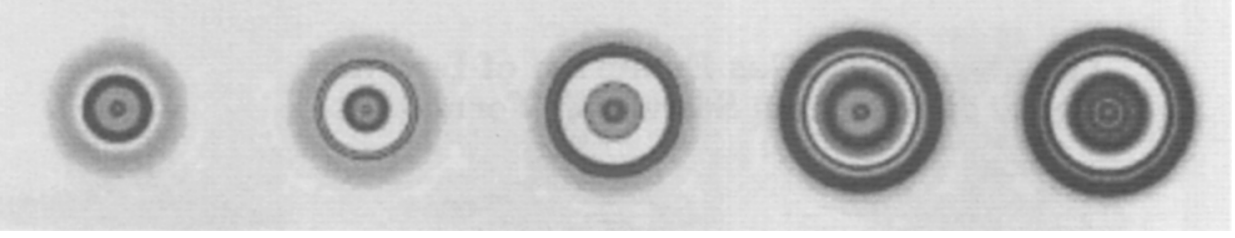

Figure 1. Logarithm of the density at time intervals of $\sim 32000 \mathrm{yr}$ during the AGB evolution of a $1 M_{\odot}$ star.

of $\sim 20000 \mathrm{yr}$. Therefore, according to our models it is not possible to use observations to recover information on the later stages of stellar evolution, such as the number of thermal pulses experienced by the star, or the time between consecutive mass-loss events. We emphasize the influence of the ISM on the deceleration and compression of the external shells. Moreover, we find that when the local ISM is taken into account, the mass of the external envelope increases appreciably due to the ISM material swept up by the wind.

We consider for the first time the effects of the AGB-PN transition phase on the PN gas structure, and find that the transition time evolution determine the size and the dynamical evolution of the PN inner shell. We study the influence of the progenitor mass on the PN observable parameters and we were able to give a logical explanation for the disagreement between the dynamical timescales in $\mathrm{PNe}$ and the evolutionary status of their central stars. We also find that, according to our models, observations severely underestimate the ionized mass present in $\mathrm{PNe}$.

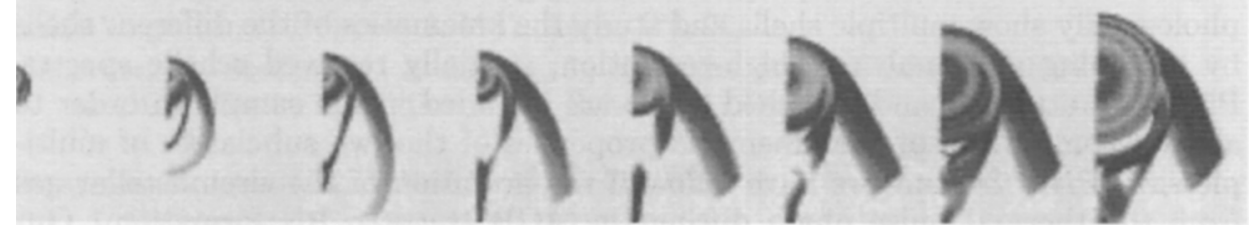

Figure 2. Density, on a logarithmic scale at time intervals of 60400 $\mathrm{yr}$, of the structure generated by a star moving with a velocity of 20 $\mathrm{km} \mathrm{s}^{-1}$ through an ISM with density $n_{0}=0.01 \mathrm{~cm}^{-3}$.

We also study the interaction of PNe with the ISM, assuming that the central star moves relative to its surroundings with a very conservative velocity $\left(20 \mathrm{~km} \mathrm{~s}^{-1}\right.$; an example is shown in Fig. 2). We find that the interaction, in contrast to what has usually been believed, has an enormous influence on the development of the PN structure from the early stages of the evolution.

Hardcopies of the thesis can be obtained by contacting: villaver@stsci.edu. 